\title{
Total and Intra-Abdominal Fat Distribution in Obese Adolescents Using Different Techniques: Anthropometry, BIA and CT
}

\author{
Soha M Abd El-Dayem¹, Sahar A El-Masry², Hamed Elghawaby³, Farid Gamil Amin, Ibrahim A Ibrahim²
}

${ }^{1}$ National Research Centre, Pediatrics Department, El-Bohooth St.,Dokki, Giza, Cairo 12622, Egypt; ${ }^{2}$ Biological Anthropology Department, Medical Research Division, National Research Centre, El-Bohooth St.,Dokki, Giza, Cairo 12622, Egypt; ${ }^{3}$ Radiology Department, Cairo University, Cairo, Egypt

\author{
Citation: El-Dayem SMA, El-Masry SA \\ Elghawaby $\mathrm{H}$, Amin FG, Ibrahim IA. Total and \\ Intra-Abdominal Fat Distribution in Obese \\ Adolescents Using Different Techniques: \\ Anthropometry, BIA and CT Maced J Med Sci. \\ Anthropometry, BIA and CT. Maced J Med Sci. \\ 10.3889/MJMS.1957-5773.2012.0237. \\ Key words: Visceral obesity; anthropometry; BIA; \\ CT; adolescents. \\ Correspondence: Dr. Sahar A El-Masry, Prof. of \\ Biological Anthropology, National Research \\ Centre, Dokki, Giza, Egypt. E-mail: \\ masrysa@yahoo.com \\ Received: 12-Jan-2012; Revised: 07-Apr-2012; \\ Accepted: 28-Apr-2012; Online first: 29-Aug-2012 \\ Copyright: $\odot 2012$ El-Dayem SMA. This is an \\ open-access article distributed under the terms of \\ the Creative Commons Attribution License, which \\ permits unrestricted use, distribution, and \\ author and source are credited. \\ Competing Interests: The author have declared \\ that no competing interests exist.
}

\section{Abstract}

Objectives: Assess the association between the anthropometric measurements of obesity, body composition with total and regional fat areas determined by CT in obese adolescents.

Patients and method: 33 obese adolescents and 20 control group were assessed using anthropometry and body composition. CT measured total abdominal, subcutaneous and visceral fat areas.

Results: Anthropometric, body composition and CT findings of fat content were significantly higher in obese adolescents. Visceral obesity estimated by $\mathrm{CT}$ in obese adolescents was significantly higher than control group $(66.7 \%$ vs. $15 \%$,). In obese subjects, total abdominal and subcutaneous fat had a significant positive correlation with BMI, waist, hip circumferences and Fat mass, while visceral fat had a significant positive correlation with total abdominal fat and waist circumference. In control subjects, total abdominal and subcutaneous fat had a significant positive correlation with BMI, waist, hip measurements, waist / hip ratio and fat free mass, while visceral fat had a significant positive correlation with $\mathrm{BMI}$, waist, hip measurements, waist/ hip ratio, FM, PBF and total abdominal fat.

Conclusion: CT is useful for diagnosis of visceral abdominal obesity in both obese and non obese subjects. Waist circumference is a good predictor of total abdominal, subcutaneous and visceral fat in obese adolescents.

\section{Introduction}

Anthropometry; such as body mass index (BMI) and waist circumference (WC); is known to be less reliable for making accurate muscle and fat mass calculations than other more recent techniques; such as body composition analyzer and CT. BMI, however, has proven to be a simple method for evaluating the existence of obesity by quickly providing results about body fat. At the same time, it has the weaknesses of being unable to distinguish between body fat and lean body mass [1].

Bioimpedance Analysis (BIA) is simple, saves time and shows high-level interrelationships with other methods of obesity assessment. However, the accuracy of BIA has yet to be proven in children and juveniles because it was originally designed for adult measurements [2].

Computed tomography (CT), magnetic resonance imaging (MRI) and dual energy X-ray absorptiometry (DEXA) are complicated and expensive methods of analyzing body fat, and require expert skills, so it is difficult to apply those methods to a wide range of people. Their accuracy for assessing body composition 
has proven to be superior to the results of anthropometry. Overall, CT seems to be the best method used in body fat research with its ability to pin-point differences between visceral fat and subcutaneous fat in the abdominal region. It is known to be the most accurate among obesity confirmation methods using direct bodily measurements [2].

So, this study was aiming to assess the association between the anthropometric measurements of obesity, body composition with total and regional fat areas determined by CT in obese adolescents.

\section{Subjects and Methods}

This cross sectional observational study was performed after obtaining approval by the ethical committee of the National Research Centre. Written informed consent was obtained from all patients, their parents and healthy non obese volunteers after full discussion about the aim of the study.

The study included 33 obese adolescents (17 boys and 16 girls) (their BMI was $>95^{\text {th }}$ percentile) and 20 age and sex matched non obese healthy volunteers as control group (12 boys and 8 girls) (their BMI was $<85^{\text {th }}$ percentile) [3]. The obese subjects were taken from the Endocrinology Clinic of the National Research Centre. The healthy non obese volunteers were taken from the pediatrics clinic in the National Research Centre who was coming for acute illness.

\section{Inclusion criteria}

1-Age more than 12 and less than 18 years.

2-Patients have exogenous obesity.

3-Weigh not more than $130 \mathrm{~kg}$ according to the criteria of the used CT machine.

\section{Exclusion criteria}

1-Patients have any endocrinal disorders confirmed by laboratory investigations and MRI done on suprarenal and pituitary area.

2- Patients have any genetic disease.

3-Patients have any chronic illness.

\section{Study Protocol}

Protocol determinations, all carried out on the same day for patients and controls, and included:
Thorough history taking including age, sex, history of any chronic illness.

\section{Anthropometric measurements}

Include weight $(\mathrm{kg})$ and height (cm). Weight was measured using a standard clinical balance (weight was approximated to the nearest $0.1 \mathrm{~kg}$ ) and height was measured using a fixed stadiometer (height was approximated to the nearest millimeter) and body mass index (BMI) $\left(\mathrm{kg} / \mathrm{m}^{2}\right)$ was calculated as the weight in $\mathrm{kg}$ divided by the height squared $\left(\mathrm{m}^{2}\right)$. Waist circumference was measured at the level of the umbilicus with the subject standing and breathing normally. Hip circumference (Hip C) at the level of the iliac crest, using non-stretchable plastic tape to the nearest $0.1 \mathrm{~cm}$. Waist / hip ratio (WHR) was calculated [4,5].

\section{Bioelectrical Impedance Analysis (BIA)}

Whole body resistance and reactance (capacitance) were measured using a Bioelectrical Impedance Analyzer (HOLTAIN LIMITED). As specified by the manufacturer, the unit was calibrated before testing using 400-ohm resistor, and electrodes were placed on right wrist and ankle with the participant sleeping on his / her back and wearing light clothing without socks. The presence of any metallic element with or beside the participant should be avoided. By using subject sex, age, weight and height approximated to the nearest unit, the percentage body fat (PBF), fat mass (FM) and fat free mass (FFM) were derived.

\section{Computed Tomography (CT)}

Abdominal, visceral and subcutaneous fat were measured using the computerized single-layer scanner, Somatom Sensation 16 (SIMENS Erlangen, Germany). Five non-enhanced images were taken from the abdominal CT scan: a scout view was taken for the whole abdomen followed by anterior 3-4 cuts at the level of the umbilicus and at the L 4-5 disc level posterior. Tracing the subcutaneous fat contour at the level of the umbilicus and calculating the attenuation range (in Hounsfield units) for fat tissue. Then, a histogram for fat tissue was computed on the basis of mean attenuation plus or minus $2 \mathrm{SD}$. From each of the images, the Total Abdominal Fat Area (TAF) and Subcutaneous Abdominal fat area was named by tracing its contour on the scan. Defining the intraperitoneal tissue by tracing its contour on the scan. Within that region of interest, tissues with attenuation values within the mean plus or minus 2 SD will be considered to be the visceral fat area. Cut off point 
El-Dayem et al. Fat Distribution in Obese Adolescents: Anthropometry, BIA and CT

of $100 \mathrm{~cm}^{2}$ of the visceral fat area estimated by CT for adults $[1,6]$ was used to classify all the subjects under study (obese and control) to know who of them had visceral obesity (more than $100 \mathrm{~cm}^{2}$ ). This cut off point was used for adults, however in this study it was used for adolescents as it was the only available one .

\section{Statistical analysis}

Statistical package for social science (SPSS) version 9.05 was used for analysis of data. Weight, height and BMI were expressed in terms of standard deviation scores (SDS) [3]. Data was tested for normal distribution using one-sample Kolomongorov-Simirnov test for total sample and subgroups, which revealed that all the data was normally distributed. So, parametric tests were used. T-test was used to compare two independent quantitative variables, while Chi-square was done to compare qualitative data. Pearson's Correlation was also done.

\section{Results}

CT findings reflected that $15 \%$ of the control group who appears healthy and non obese; suffering from visceral obesity (> $100 \mathrm{~cm}^{2}$ ), while $66.7 \%$ of the obese group suffering from visceral obesity. Anthropometric, body composition and CT findings showed insignificant sex difference in both obese and control groups $(p>0.05)$. So, the analysis was completed without sex differentiation.

Anthropometric (BMI, waist, hip and waist / hip ratio), body composition (FM, FFM and PBF) and CT finding (total abdominal, subcutaneous and visceral fat)

Table 1: Comparisons between anthropometric measurements, body composition and CT findings of the obese and control adolescents groups.

\begin{tabular}{|c|c|c|c|c|c|c|}
\hline & \multicolumn{2}{|c|}{ Obese (33) } & \multicolumn{2}{|c|}{ CONTROL (20) } & \multirow[b]{2}{*}{$\mathrm{t}$} & \multirow[b]{2}{*}{ P-value } \\
\hline & MEAN & S.D & MEAN & S.D & & \\
\hline \multicolumn{7}{|l|}{ Anthropometric measurements : } \\
\hline WEIGHT FOR AGE (SDS) & 1.654 & 1.333 & 0.765 & 1.959 & 1.967 & 0.055 \\
\hline HEIGHT FOR AGE (SDS) & -0.550 & 1.120 & -0.215 & 1.025 & -1.115 & 0.271 \\
\hline BMI (SDS) & 2.559 & 2.515 & 1.057 & 1.875 & 2.307 & 0.025 \\
\hline WAIST Circumference $(\mathrm{cm})$ & 101.06 & 12.22 & 74.5 & 10.06 & 8.18 & 0.000 \\
\hline HIP circumference $(\mathrm{cm})$ & 110.42 & 12.15 & 85.8 & 8.12 & 8.03 & 0.000 \\
\hline WAIST/HIP RATIO & 0.916 & 5.481 & 0.866 & 4.876 & 3.333 & 0.002 \\
\hline \multicolumn{7}{|l|}{ Body composition: } \\
\hline FM (Kg) & 34.59 & 15.07 & 10.25 & 5.61 & 6.92 & 0.000 \\
\hline FFM $(\mathrm{Kg})$ & 46.02 & 11.45 & 40.00 & 8.37 & 2.04 & 0.047 \\
\hline PBF (\%) & 42.09 & 12.36 & 19.86 & 8.20 & 7.14 & 0.000 \\
\hline \multicolumn{7}{|l|}{ CT findings (Areas $\mathrm{cm}^{2}$ ): } \\
\hline TOTAL ABDOMINAL FAT & 671.88 & 180.03 & 363.87 & 88.79 & 7.13 & 0.000 \\
\hline SUBCUTANEOUS FAT & 404.75 & 159.40 & 164.09 & 100.90 & 6.05 & 0.000 \\
\hline VISCERAL FAT & 120.53 & 33.98 & 56.52 & 46.37 & 5.78 & 0.000 \\
\hline
\end{tabular}

were significantly higher in obese adolescents than controls (Table1).

Table 2: Pearson's correlation between CT findings and the anthropometric measurements and body composition parameters of obese adolescent group.

\begin{tabular}{|c|c|c|c|c|}
\hline \multirow{2}{*}{ Variables } & & \multicolumn{3}{|c|}{ CT findings } \\
\hline & & Total abdominal fat & Subcutaneous fat & Visceral fat \\
\hline \multirow{2}{*}{ WEIGHT (SDS) } & $r$ & $0.794^{\star \star}$ & $0.722^{\star *}$ & 0.321 \\
\hline & $P$-value & 0.000 & 0.000 & 0.069 \\
\hline \multirow{2}{*}{ HEIGHT (SDS) } & $\mathrm{r}$ & 0.340 & 0.332 & 0.124 \\
\hline & $P$-value & 0.053 & 0.059 & 0.490 \\
\hline \multirow{2}{*}{ BMI (SDS) } & $\mathrm{r}$ & $0.745^{\star \star}$ & $0.662^{* *}$ & 0.316 \\
\hline & $P$-value & 0.000 & 0.000 & 0.073 \\
\hline \multirow{2}{*}{ WAIST (cm) } & $\mathrm{r}$ & $0.870^{* *}$ & $0.874^{* *}$ & $0.347^{*}$ \\
\hline & $P$-value & 0.000 & 0.000 & 0.048 \\
\hline \multirow{2}{*}{ HIP cm) } & $\mathrm{r}$ & $0.887^{* *}$ & $0.873^{* *}$ & 0.336 \\
\hline & $P$-value & 0.000 & 0.000 & 0.056 \\
\hline \multirow{2}{*}{ WAIST/HIP Ratio } & $\mathrm{r}$ & 0.132 & 0.171 & 0.052 \\
\hline & $P$-value & 0.465 & 0.341 & 0.772 \\
\hline \multirow{2}{*}{ FM (kg) } & $\mathrm{r}$ & $0.729^{* *}$ & $0.634^{* *}$ & 0.224 \\
\hline & $P$-value & 0.000 & 0.000 & 0.210 \\
\hline \multirow{2}{*}{ FFM (kg) } & $\mathrm{r}$ & 0.277 & 0.289 & 0.204 \\
\hline & $P$-value & 0.118 & 0.102 & 0.254 \\
\hline \multirow{2}{*}{ PBF (\%) } & $\mathrm{r}$ & $0.416^{*}$ & 0.332 & 0.105 \\
\hline & $P$-value & 0.016 & 0.059 & 0.560 \\
\hline \multirow[t]{2}{*}{ Total abdominal fat $\left(\mathrm{cm}^{2}\right)$} & $\mathrm{r}$ & & & 0.4 \\
\hline & $P$-value & & & $0.01^{*}$ \\
\hline \multirow[t]{2}{*}{ Subcutaneous fat $\left(\mathrm{cm}^{2}\right)$} & $\mathrm{r}$ & & & 0.3 \\
\hline & $P$-value & & & 0.09 \\
\hline
\end{tabular}

$\mathrm{FFM}=$ fat free mass; $\mathrm{PBF}=$ percentage body fat; $\mathrm{CT}=$ Computed tomography.

Total abdominal and subcutaneous fat areas had a significant positive correlation with body weight, BMI, waist, hip measurements and FM. Total abdominal fat had a significant positive correlation with PBF while, visceral fat had a significant positive correlation with total abdominal fat and waist in obese group (Table 2).

Table 3: Pearson's correlation between CT findings and the anthropometric and body composition parameters of the control adolescents group

\begin{tabular}{|c|c|c|c|c|}
\hline \multirow[t]{2}{*}{ Variables } & & \multicolumn{3}{|c|}{ CT findings } \\
\hline & & Total abdominal fat & Subcutaneous fat & Visceral fat \\
\hline \multirow{2}{*}{ WEIGHT (SDS) } & $r$ & $0.826^{* *}$ & $0.741^{* *}$ & $0.505^{*}$ \\
\hline & $P$-value & 0.000 & 0.000 & 0.023 \\
\hline \multirow{2}{*}{ HEIGHT (SDS) } & $\mathrm{r}$ & $0.581^{\star \star}$ & $0.586^{* \star}$ & 0.216 \\
\hline & $P$-value & 0.007 & 0.007 & 0.360 \\
\hline \multirow{2}{*}{ BMI (SDS) } & $\mathrm{r}$ & $0.548^{*}$ & 0.416 & $0.477^{\star}$ \\
\hline & $P$-value & 0.012 & 0.068 & 0.034 \\
\hline \multirow{2}{*}{ WAIST (cm) } & $\mathrm{r}$ & $0.809^{* *}$ & $0.694^{* *}$ & $0.543^{*}$ \\
\hline & $P$-value & 0.000 & 0.001 & 0.013 \\
\hline \multirow{2}{*}{ HIP (cm) } & $\mathrm{r}$ & $0.699^{* *}$ & $0.594^{* *}$ & $0.476^{*}$ \\
\hline & $P$-value & 0.001 & 0.006 & 0.034 \\
\hline \multirow{2}{*}{ WAIST/HIP Ratio } & $\mathrm{r}$ & $0.750^{* *}$ & $0.662^{\star *}$ & $0.487^{*}$ \\
\hline & $P$-value & 0.000 & 0.001 & 0.029 \\
\hline \multirow{2}{*}{ FM (kg) } & $\mathrm{r}$ & $0.535^{\star}$ & 0.266 & $0.600^{\star *}$ \\
\hline & $P$-value & 0.015 & 0.257 & 0.005 \\
\hline \multirow{2}{*}{ FFM (kg) } & $\mathrm{r}$ & $0.730^{\star \star}$ & $0.798^{\star \star *}$ & 0.263 \\
\hline & $P$-value & 0.000 & 0.000 & 0.262 \\
\hline \multirow{2}{*}{ PBF (\%) } & $\mathrm{r}$ & 0.272 & -0.011 & $0.499^{*}$ \\
\hline & $P$-value & 0.247 & 0.965 & 0.025 \\
\hline \multirow[t]{2}{*}{ Total abdominal fat $\left(\mathrm{cm}^{2}\right)$} & $\mathrm{r}$ & & & 0.7 \\
\hline & $P$-value & & & $0.001^{*}$ \\
\hline \multirow[t]{2}{*}{ Subcutaneous fat $\left(\mathrm{cm}^{2}\right)$} & $\mathrm{r}$ & & & 0.3 \\
\hline & $P$-value & & & 0.2 \\
\hline
\end{tabular}

$\mathrm{FFM}=$ fat free mass; PBF = percentage body fat; $\mathrm{CT}$ = Computed tomography. 
On the other hand, total abdominal and subcutaneous fat areas had a significant positive correlation with body weight, BMI, waist, hip measurements, waist/hip ratio and FFM. Total abdominal fat had a significant positive correlation with FM while, visceral fat had a significant positive correlation with $\mathrm{BMI}$, waist, hip measurements, waist/ hip ratio , FM, PBF and total abdominal fat in controls (Table 3).

\section{Discussion}

In the present study, Anthropometric (BMI, waist, hip and waist / hip ratio), body composition (FM, FFM and PBF) and CT finding (total abdominal, subcutaneous and visceral fat) were significantly higher in obese adolescents than controls.

This coincides with the study of Kamel et al [7], who found that waist circumference, WHR and fat mass were higher in Egyptian prepubertal obese children than in normal control.

Berker et al., [1] reported that people with $>100$ $\mathrm{cm}^{2}$ CT-determined visceral fat area, which is called visceral obesity, have higher rates of diabetes mellitus and coronary artery disease. The present study revealed that Visceral obesity estimated by CT in obese children was significantly higher than control group (66.7\% vs 15 $\%, \mathrm{P}$ value $\left.=0.0001^{*}\right)$.

Anthropometric measurements have been widely used to assess obese children [8] being very simple and cheap but they are not very accurate and the reference values have varied a lot and they cannot differentiate between subcutaneous and intra-abdominal visceral fat [2]. BMI being one of the important anthropometric indices reflects both fat and fat free components of the body weight, without differentiation between them. The increase in BMI is either accompanied by increase in the fat mass and concomitant decrease in the fat free mass or the reverse. Even within the normal range of $\mathrm{BMI}$, accumulation of visceral fat remains an independent cardiovascular risk factor. This observation led researchers and clinicians alike to believe that clinical diagnosis of visceral adiposity may be more important than the current diagnosis of obesity using the BMI [9].

In this study, comparison between anthropometric characteristics, body composition, and $\mathrm{CT}$ findings of the obese and control groups by sex revealed insignificant sex differences in all the parameters in this study. This coincides with the findings of Furukawa et al. [10] in Japan and Berker et al. [1] in Turkey. In Korea, Yu et al. [2] found that, although the average body FM of overweight elementary school boys was higher than that of girls and the average FFM of girls was higher than that of boys, the differences were insignificant.

In the current study, total abdominal and subcutaneous fat areas have a significant positive correlation with BMI, WC, HC and FM in obese children and have a significant positive correlation with WC, HC, WHR and FFM in the controls.

In Southern Siri-Lanka; Weerarathna et al [11], stated that total abdominal fat showed positive correlations with BMI, WC, HC and WHR. While the findings of Hsieh and Muto [12], indicated that utility of WC as a marker of adiposity and cardiovascular risk factor can be improved by relating it to height and not to hip circumference.

These agree with several studies which show that WC is strongly correlated with total abdominal fat, subcutaneous abdominal fat and truncal fat estimated by $C T$ at the level of umbilicus $[2,11,13,14,15]$. Although there are many studies using CT for adults, the studies for children are very little.

In our study, visceral fat had a significant positive correlation with WC and total abdominal fat in obese children.

In clinical practice, the WC and WHR are the commonly used anthropometric measures to diagnose abdominal obesity. These measures were found to correlate with total amount of visceral fat measured by abdominal CT scanning [9]. In adults, waist circumference of equal or greater than $102 \mathrm{~cm}$ (> $40 \mathrm{inch}$ ) in men or 88 $\mathrm{cm}(>35 \mathrm{inch})$ in women was used as cut off. These widely used cut-points were originally based on a replacement of the classification of BMI, but other cutpoints have also been suggested on the basis of relationships with visceral fat area [9]. However, there is no universally accepted cut off points for the WC in children.

The WC and WHR are important prognostic indicators of the metabolic disease [11]. Because the WC alone are more strongly correlated with visceral fat than the WHR, guidelines tend to focus on WC to estimate disease risk. Association of WC with cardiovascular diseases is well recognized. In a major diabetes prevention study, WC was the best predictor of development of diabetes among individuals with impaired glucose tolerance [1]. In the WHO MONICA study, the 
degree of overweight was reflected by the WC and not by the WHR. The WC has better sensitivity and utility over WHR as an anthropometric measurement of obesity [10].

Abdominal obesity is usually assessed by the easily measured WC, WHR, or the less-commonly used sagittal abdominal diameter (SAD). By the use of sophisticated imaging techniques, such as MRI and CT, different fat depots can be distinguished at the waist level, in particular the visceral fat depot which is associated with increased risk of cardio metabolic complications [16]. CT scan is considered the most accurate way to assess obesity and differentiate between subcutaneous and intra-abdominal visceral fat $[7,17]$.

In the contrary, Hamdy et al, [9] and Yu et al [2] reported that visceral obesity by $\mathrm{CT}$ was not correlated with the waist circumference, measured WHR and body fat content estimated by BIA.

Berker et al, [1] concluded that Anthropometric measurements and visceral fat tissue measurement methods such as Ultrasound and BIA exhibit differences with respect to compliance with $\mathrm{CT}$ results in visceral fat tissue measurements by gender and BMI levels (obese and non obese).

In our study, visceral fat had a significant positive correlation with BMI, WC, HC, WHR , FM, PBF and total abdominal fat in controls. BMI together with WC and WHR are anthropometric parameters commonly used for the prediction of intra-abdominal fat deposition. While methods such as BMI and anthropometric parameters can predict the amount of visceral adipose tissue, they become inadequate as BMI increases [1].

Conclusion: That, CT is useful for diagnosis of visceral abdominal obesity. Waist circumference is a good predictor of total abdominal, subcutaneous and visceral fat in obese adolescents. Visceral fat is significantly higher in obese adolescents.

Recommendation: Measurement of visceral obesity by CT at the level of the umbilicus; during exclusion of the Cushing syndrome; may identify adolescents at risk of metabolic complications who may benefit from further assessment or interventions. A standard cut off of WC in children and adolescents must be done as it is a better indicator of visceral abdominal fat than BMI.

Limitation of the study: 1) CT is expensive; 2) Exposure to ionizing radiation of $\mathrm{CT} ; 3$ ) Difficulty of administration of CT; 4) No cut off level of waist circumference is available for children and adolescence; 5) Small number of cases.

\section{Acknowledgements}

We would like to acknowledge all the adolescents who participated in this study and their parents, the technicians who helped in the CT assessment and the doctors who participated in collection of the data. Without their help, this study couldn't have been completed.

\section{Statement of authorship}

All authors participated in the conception and design of the study, drafting the article and final approval of the version to be submitted. S El-Dayem was responsible for collection of obese subjects and exclusion of presence of any endocrinal disease. S El-Masry was responsible for analysis and interpretation of the data. $\mathrm{HG}$ was responsible for $\mathrm{Ct}$ assessment. FA was responsible for revising the article for important intellectual content. I.I. was responsible for collection of data and scientific references, share in drafting the article. All authors read and approved the final manuscript.

\section{References}

1. Berker D, Koparal S, Ip ik S, Pap aoðlu L, Aydin Y, Erol K, Delibap i T, Güler S. Compatibility of different methods for the measurement of visceral fat in different body mass index strata. Diagn Interv Radiol. 2010;16(2):99-105.

2. Yu OK, Rhee YK, Park TS, Cha YS. Comparisons of obesity assessments in over-weight elementary students using anthropometry, BIA, CT and DEXA. Nutr Res Pract. 2010; 4(2):128-35.

3. Ghalli I, Salah N, Hussien F, Erfan M, El-Ruby M, Mazen I, Sabry M, Abd El-Razik M, Saad M, Hossney S, Ismaail and Abd El-Dayem S. Egyptian growth curves for infants, children and adolescents. Published in: Crecere nel mondo. Satorio A, Buckler JMH and Marazzi N ,2008, Ferring Publisher, Italy, 2002.

4. Hiernaux J. and Tanner J.M. Growth and physical studies. In: Human Biology: A guide to field methods. 2nd Eds. Pp. 213. Edited by Weiner J.S., Lourie S.A., IBP. London, Blackwell Scientific Publications. Oxford. U.K., 1969.

5. Cameron N. The methods of auxological anthropology in: Falkner F, Tanner JM. Human Growth: A Comprehensive Treatise. 2nd Ed. New York, NY: Plenum press, 1986. 
6. Lee YH, Lee SH, Jung ES, Kim JS, Shim CY, Ko YG, Choi $D$, Jang Y, Chung N, Ha JW. Visceral adiposity and the severity of coronary artery disease in middle-aged subjects with normal waist circumference and its relation with lipocalin-2 and MCP1. Atherosclerosis. 2010;213(2):592-7.

7. Kamel AF, Salah Eldin N, Mourad A, Badawy N, Hegazy H. Obesity and Growth Hormone Deficiency: A Study of Body Fat Distribution. Research Journal of Medicine and Medical Sciences. 2009;4(2):326-331.

8. Kullberg J, Below CV, Lönn L, Lind L, Ahlstrom H, Johansson L. Practical approach for estimation of subcutaneous and visceral adipose tissue. Clin Physiol Funct Imaging. 2007; 27:148-153.

9. Hamdy O, Porramatikul S, Al-Ozairi E. Metabolic obesity: the paradox between visceral and subcutaneous fat. Curr Diabetes Rev. 2006;2(4):367-73.

10. Furukawa K, Katabami T, Nakajima $Y$, Sato $T$, Kato $H$, Koganei R, et al. Evaluation of whole-abdominal fat volume by 700 -slice CT scanning and comparison with the umbilical fat area anthropometric indices. Obes Res Clin Pract. 2010; 4:e111-e117.

11. Weerarathna TP, Lekamwasam S, Rodrigo M. Prediction of total and visceral fat contents using anthropometric measures of adiposity in women. Ceylon Med J. 2008; 53(4):128-32.
12. Hsieh SD, Muto T. Metabolic syndrome in Japanese men and women with special reference to the anthropometric criteria for the assessment of obesity: proposal to use the waist-toheight ratio. Preventive Medicine. 2006;42:135-9.

13. Shin YH. A review of childhood obesity. Journal of Korean Academy of Child Health Nursing. 2005;11:240-5.

14. Thang SH, Naveed S, Mike L. ABC of obesity: assessment of obesity and its clinical implications. British Medical Journal. 2006;30:695-8.

15. Sampaio LR, Simões EJ, Assis AM, Ramos LR. Validity and reliability of the sagittal abdominal diameter as a predictor of visceral abdominal fat. Arq Bras Endocrinol Metabol. 2007; 51(6):980-6.

16. Snijder MB, Van-Dam RM, Visser M, Seidell JC. What aspects of body fat are particularly hazardous and how do we measure them? International Journal of Epidemiology. 2006; 35: 83-92.

17. Seo JA, Kim BG, Cho H, Kim HS, Park J, Baik SH, Choi DS, Park MH, Jo SA, Koh YH, Han C, Kim NH. The cut off values of visceral fat area and waist circumference for identifying subjects at risk for metabolic syndrome in elderly Korean: Ansan Geriatric (AGE) cohort study. BMC Public Health. 2009;9:443. 